\title{
Epidemiology of Rotavirus and Cholera in Children Aged Less Than Five Years in Rural Bangladesh
}

\author{
A.K. Siddique', Sirajuddin Ahmed', Anwarul lqbal', Arif Sobhan', Goutam Poddar', \\ Tasnim Azim', D.A. Sack², Mustafizur Rahman', and R.B. Sack ${ }^{2}$ \\ 'ICDDR,B, GPO Box 128, Dhaka 1000, Bangladesh and 2Johns Hopkins Bloomberg School of Public Health, 615 North Wolfe \\ Street, Baltimore, MD 21205, USA
}

\begin{abstract}
Despite the known presence of rotavirus-associated diarrhoea in Bangladesh, its prevalence, including records of hospitalization in rural health facilities, is largely unknown. In a systematic surveillance undertaken in two government-run rural health facilities, 457 children, aged less than five years, having acute watery diarrhoea, were studied between August 2005 and July 2007 to determine the prevalence of rotavirus. Due to limited financial support, the surveillance of rotavirus was included as an addendum to an ongoing study for cholera in the same area. Rotavirus infection was detected in 114 (25\%) and Vibrio cholerae in 63 (14\%) children. Neither rotavirus nor V. cholerae was detected in 280 (61\%) samples; these were termed 'non-rotavirus and non-cholera' diarrhoea. Both rotavirus and cholera were detected in all groups of patients (<5 years). The highest proportion ( $41 \% ; 47 / 114)$ of rotavirus was in the age-group of $6-11$ months. In children aged less than 18 months, the proportion $(67 \% ; 76 / 114)$ of rotavirus was significantly $(\mathrm{p}<0.001)$ higher than that of cholera $(16 \% ; 10 / 63)$. By contrast, the proportion $(84 \% ; 53 / 63)$ of cholera was significantly $(\mathrm{p}<0.001)$ higher than that of rotavirus $(33 \%$; 38/114) in the age-group of 18-59 months. During the study period, 528 children were hospitalized for various illnesses. Thirty-eight percent $(202 / 528)$ of the hospitalizations were due to acute watery diarrhoea, and $62 \%$ were due to non-diarrhoeal illnesses. Rotavirus accounted for $34 \%$ of hospitalizations due to diarrhoea. Severe dehydration was detected in $16 \%$ (74/457) of the children. The proportion $(51 \% ; 32 / 63)$ of severe dehydration among $V$. cholerae-infected children was significantly higher $(\mathrm{p}<0.001)$ compared to the proportion $(16 \% ; 18 / 114)$ of rotavirus-infected children. The study revealed that $12-14 \%$ of the hospitalizations in rural Bangladesh in this age-group were due to rotavirus infection, which has not been previously documented.
\end{abstract}

Key words: Cholera; Diarrhoea; Hospitalizations; Rotavirus infections; Vibrio cholerae; Bangladesh

\section{INTRODUCTION}

Rotavirus is the most common cause of diarrhoea in infants and young children in both developed and developing countries (1). On a global scale, rotavirus is responsible for nearly 140 million cases of diarrhoea each year in children aged six months to two years $(2,3)$. It is also one of the major contributors to deaths of infants and young children in developing countries (2), claiming 500,000-600,000 lives each year (4-6), and up to $85 \%$ of these deaths

Correspondence and reprint requests should be addressed to:

Dr. A.K. Siddique

Public Health Sciences Division

ICDDR,B

GPO Box 128, Dhaka 1000

Bangladesh

Email: siddique@icddrb.org

Fax: 880-2-8826050 occur in low-income countries (4). Diarrhoea due to rotavirus in children is recognized as the leading cause of hospitalizations worldwide (7).

The disease affects infants and children aged three months and by age 3-4 years; virtually all children have had the disease. Previous infection offers protection from subsequent illnesses. However, re-infections are frequent but subsequent illnesses tend to be less severe than the first infection (8). Many rotavirus infections are asymptomatic, particularly among infants aged less than three months, older children, and adults $(9,10)$. Immunity from repeated exposures to rotavirus probably accounts for the high infection-to-illness ratio among older children and adults. Rotavirus infections are likely to be associated with dehydration $(11,12)$. The majority of deaths from diarrhoea due to rotavirus occur in Africa, the Indian Sub-continent, and Latin Americas. Despite the widespread recognizable prevalence of 
diarrhoea due to rotavirus in Asia, including the Indian Sub-continent and the Asia-Pacific region, information on the incidence and the disease is grossly deficient $(13,14)$. Hospitalization data seem to be scantier than incidence data.

Diarrhoeal disease is one of the major public-health problems in Bangladesh. For nearly two decades, it has been recognized that rotavirus is one of the common causes of diarrhoea in children in urban Bangladesh. A surveillance conducted in the late nineties at the ICDDR,B hospital in urban Dhaka suggested that $20 \%$ of children seeking treatment in this diarrhoea-treatment facility suffer from rotavirus infection (15). Little is known about the epidemiology of diarrhoea due to rotavirus in rural Bangladesh where about $90 \%$ of the population lives. We also do not know much about the magnitude of hospitalization due to rotavirus, particularly in rural health facilities. Results of a recent study in Matlab, where the International Centre for Diarrhoeal Disease Research, Bangladesh (ICDDR,B) maintains a Health and Demographic Surveillance System and a diarrhoea-treatment centre, showed that rotavirus was a major cause of hospitalizations in this treatment centre (16).

This paper presents results of the two-year clinical surveillance of rotavirus-associated diarrhoea involving two rural areas of Bangladesh. The surveillance was undertaken to study the epidemiology of diarrhoea due to rotavirus among children aged less than five years (under-five children) and to assess the rate of hospitalizations due to rotavirus-associated diarrhoea at rural health facilities in Bangladesh. The health facilities where the surveillance of rotavirus was conducted were also the sites for an ongoing epidemiological and ecological study for cholera supported by a separate grant. Adding the specific requirements for the rotavirus surveillance study as an addendum to the cholera protocol rendered the rotavirus study feasible within the limits of financial support offered for the purpose.

\section{MATERIALS AND METHODS}

\section{Clinical surveillance}

The clinical surveillance was conducted in Bakerganj and Mathbaria upazilas (subdistricts), each with a population of $\sim 250,000$. Bakerganj is located in the southern region of Bangladesh, $300 \mathrm{~km}$ from the capital city Dhaka. Mathbaria is situated in the southern-most region of the country, close to mangrove swamps of the Sunderbans, at a distance of over $350 \mathrm{~km}$ from Dhaka city. The facility-based clinical surveillance was conducted in Bakerganj and Mathbaria Upazila Health Complexes (UHCs) which are grassroots-level rural hospitals run by the national health services of the country. Each hospital has 31-bed inpatient-care facility. These hospitals also offer outpatient care and other preventive care services. The UHC is also the referral centre for a number of grassroots-level community clinics.

The method of this surveillance system has previously been described (17). In short, the clinical surveillance was conducted every 15 days between August 2005 and July 2007. A study physician who visited the surveillance site stayed there for three days during which time he personally observed all patients presenting with acute watery diarrhoea. The study physician administered a pre-coded questionnaire and recorded demographic information, history of the onset of illness, and clinical findings. A detailed physical examination for each patient was carried out. The dehydration status of patients was determined objectively using the criteria of the World Health Organization (WHO) and was categorized as 'no detectable dehydration', 'some dehydration', or 'severe dehydration' (18). Standard therapy (rehydration, nutritional support, and antibiotics when necessary) for diarrhoeal illness was given. During the three-day surveillance period, the study physician also collected clinical parameters of all under-five children admitted for inpatient care by the hospital physicians for all types of illness, including acute watery diarrhoea.

A case of rotavirus-associated diarrhoea was defined as an under-five child seeking treatment for diarrhoea at the outpatient or hospitalized in whose stool the presence of rotavirus was demonstrated by means of enzyme immunoassay (19). In this study, no attempt was made to identify pathogens other than rotavirus and cholera associated with watery diarrhoea.

For comparison, this report also includes data of patients infected with $V$. cholerae to determine the severity of dehydration and impact on hospitalization due to rotavirus infection in under-five children.

Data were analyzed using the Epi Info software (version 3.3.2), and the $\chi^{2}$-test for difference was used in this study.

\section{Laboratory methods}

Stool specimens were obtained from each patient seen by the study physician for laboratory identification of rotavirus and placed in a sterile screw-cap container. Rectal swabs, collected from all patients, were placed in the Cary-Blair medium for culture of $V$. cholerae. All the specimens were properly labelled with information, including a unique identification 
number and the date of collection. Specimens were stored temporarily in refrigerators at $4-8{ }^{\circ} \mathrm{C}$ before transporting to the ICDDR,B laboratory in Dhaka in cold-boxes with ice-packs.

At the ICDDR,B laboratory, rotavirus antigens (group A rotavirus-specific VP6 proteins) were detected in stool specimens using a solid-phase sandwich-type enzyme immunoassay modelled after the Dakopatts commercial kit (Dakopatts, Copenhagen, Denmark), incorporating rabbit hyperimmune antisera produced at ICDDR,B and an anti-human rotavirus horseradish conjugate (2). A multiplex reverse transcriptase-polymerase chain reaction (RT-PCR) was performed on rotavirus-positive stool samples for rotavirus $\mathrm{G}$ and $\mathrm{P}$ genotyping using type-specific oligonucleotide primers (2). For the identification of $V$. cholerae, all the rectal swabs were cultured using standard bacteriological methods (20). Swabs were cultured both directly and after six-hour enrichment in alkaline peptone water on selective media at $37{ }^{\circ} \mathrm{C}$. Suspected colonies resembling $V$. cholerae were agglutinated with antisera specific for $V$. cholerae $\mathrm{O} 1$ and $V$. cholerae $\mathrm{O} 139$.

\section{Ethics}

Informed consent was obtained from parents accompanying the children. The Research Review Committee and the Ethical Review Committee of ICDDR,B and the Institutional Review Board of the Johns Hopkins Bloomberg School of Public Health approved the research protocol.

\section{RESULTS}

The systematic surveillance was conducted between August 2005 and July 2007 in the two surveillance sites. During the period, 457 under-five children with acute watery diarrhoea from both outpatient and inpatient departments were studied. Laboratory examination revealed that 114 (25\%) samples were
For the purpose of this study, under-five patients were categorized in five different age-groups. The figure shows the distribution of age-specific illness due to rotavirus-associated diarrhoea, cholera, and 'non-rotavirus and non-cholera' diarrhoea. Both rotavirus infection and cholera were detected in all the age-groups. The highest age-group frequency $(41 \% ; 47 / 114)$ of rotavirus was noted in the agegroup of 6-11 months. This was much higher than in cholera and 'non-rotavirus and non-cholera' diarrhoea patients in this age-group. Further analysis of the age-specific distribution between rotavirus and cholera in children aged less than 18 months revealed that the proportion $(67 \% ; 76 / 114)$ of rotavirus infection was significantly $(\mathrm{p}<0.001)$ higher than the proportion $(16 \% ; 10 / 63)$ of cholera infection. By contrasts, in children aged 18-59 months, the proportion $(84 \% ; 53 / 63)$ of $V$. cholerae-associated infection was significantly $(\mathrm{p}<0.001)$ higher than that of rotavirus $(33 \% ; 38 / 114)$. It was also revealed that $77 \%$ of the children were rotavirusinfected by the time they were aged 24 months.

During the surveillance period, 528 under-five children were hospitalized with various illnesses (Table 2) in the two surveillance hospitals (Bakerganj-256 and Mathbaria-272). The hospitalized cases of under-five children were divided into (a) non-diarrhoea cases (326/528) and (b) acute watery diarrhoea cases (202/528). This was done based on the observation that acute watery diarrhoea was the cause of all hospitalized diarrhoea patients in this age-group. Acute watery diarrhoea accounted for $38 \%(202 / 528)$ of hospital admissions compared to non-diarrhoea patients $(62 \% ; 326 / 528)$. Further analysis of the hospitalized cases of acute watery diarrhoea revealed that rotavirus infection accounted for 34\% (69/202) of the admitted cases compared to cholera (25\%; 50/202). The 'non-rotavirus and non-cholera' watery diarrhoea was responsible for $41 \%(83 / 202)$ of all diarrhoeal admissions. The

\begin{tabular}{|c|c|c|c|c|c|c|c|}
\hline \multirow{3}{*}{$\begin{array}{l}\text { Surveillance } \\
\text { site }\end{array}$} & \multirow{3}{*}{$\begin{array}{l}\text { No. of acute } \\
\text { watery diar- } \\
\text { rhoea cases }\end{array}$} & \multicolumn{4}{|c|}{ No. positive } & \multirow{2}{*}{\multicolumn{2}{|c|}{$\begin{array}{l}\text { Non-rotavirus and non- } \\
\text { cholera cases }\end{array}$}} \\
\hline & & \multicolumn{2}{|c|}{ Rotavirus } & \multicolumn{2}{|c|}{ V. cholerae } & & \\
\hline & & No. & $\%$ & No. & $\%$ & No. & $\%$ \\
\hline Bakerganj & 197 & 45 & 22.8 & 37 & 18.8 & 115 & 58.4 \\
\hline Mathbaria & 260 & 69 & 26.5 & 26 & 10.0 & 165 & 63.5 \\
\hline Total & 457 & 114 & 24.9 & 63 & 13.8 & 280 & 61.3 \\
\hline
\end{tabular}

positive for rotavirus antigen; $V$. cholerae was detected in 63 samples (14\%); and in 280 (61\%) samples, neither rotavirus nor $V$. cholerae was detected (Table 1 ). Cases of mixed infection with rotavirus and $V$. cholerae were not observed during the study period. highest proportion (42\%) of 69 children hospitalized with rotavirus were aged 6-11 months. It was observed that $13 \%$ (average of the two sites) of all hospitalizations in our surveillance sites (12\% in Bakerganj and 14\% in Mathbaria) were due to rota- 
Fig. Percent distribution of study children by aetiologic agent and age-group in Bakerganj and Mathbaria, Bangladesh, August 2005-July 2007

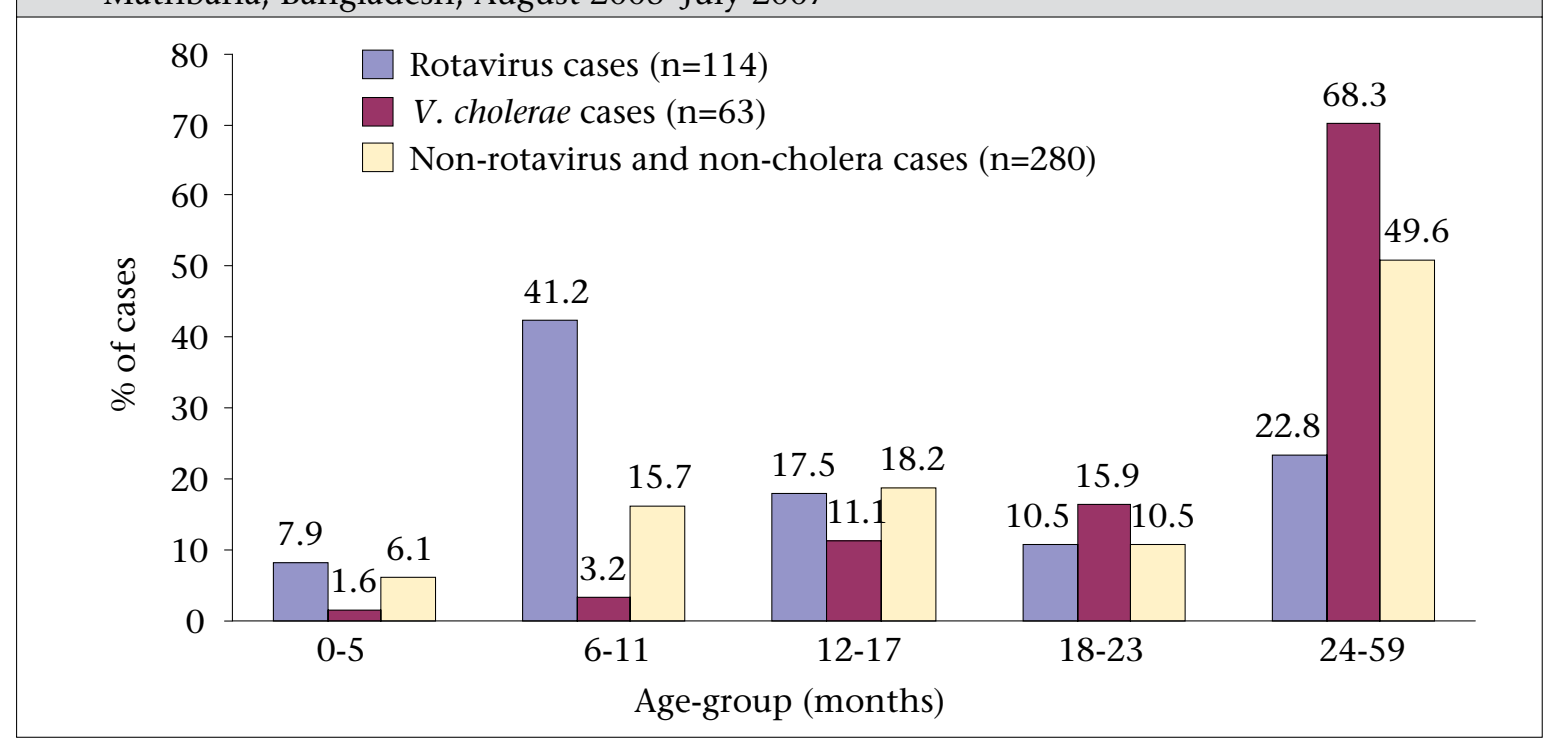

virus infection. Cholera was responsible for $9.5 \%$ of the total admissions. It was also revealed that $16 \%$ tion status revealed that most rotavirus-infected children had signs of some dehydration, which

\begin{tabular}{|c|c|c|c|c|c|c|c|c|c|}
\hline \multirow{3}{*}{$\begin{array}{l}\text { Surveillance } \\
\text { site }\end{array}$} & \multicolumn{8}{|c|}{ Diarrhoea cases } & \multirow{3}{*}{ Total } \\
\hline & \multicolumn{2}{|c|}{$\begin{array}{l}\text { Non-diarrhoea } \\
\text { cases }\end{array}$} & \multicolumn{2}{|c|}{$\begin{array}{l}\text { Rotavirus } \\
\text { diarrhoea }\end{array}$} & \multicolumn{2}{|c|}{ Cholera } & \multicolumn{2}{|c|}{$\begin{array}{c}\text { Non-rotavirus and } \\
\text { non-cholera diarrhoea }\end{array}$} & \\
\hline & No. & $\%$ & No. & $\%$ & No. & $\%$ & No. & $\%$ & \\
\hline Bakerganj & 163 & 63.7 & 31 & 12.1 & 27 & 10.5 & 35 & 13.7 & 256 \\
\hline Mathbaria & 163 & 60.0 & 38 & 14.0 & 23 & 8.5 & 48 & 17.7 & 272 \\
\hline Total & 326 & 61.7 & 69 & 13.1 & 50 & 9.5 & 83 & 15.7 & 528 \\
\hline
\end{tabular}

of all hospitalizations were due to 'non-rotavirus and non-cholera' diarrhoea.

Analysis of the overall dehydration status of the 457 under-five patients with acute watery diarrhoea observed by us revealed that 250 (55\%) had no detectable signs of dehydration. One hundred and thirty-three (29\%) children had signs of some dehydration, and severe dehydration was observed in $74(16 \%)$ cases. Further analysis of the dehydra- was significantly higher than cholera cases (47\%; 54/114 vs 30\%; 19/63; $\mathrm{p}<0.05$ ) and 'non-rotavirus and non-cholera' diarrhoea cases (47\%; 54/114 vs $21 \% ; 60 / 280 ; \mathrm{p}<0.001)$. In contrast, the proportion $(51 \% ; 32 / 63)$ of severe dehydration among the cholera patients was significantly $(\mathrm{p}<0.001)$ higher than among the rotavirus-infected patients (16\%; $18 / 114$ ) and 'non-rotavirus and non-cholera' diarrhoea patients $(9 \% ; 24 / 280)$. It was also noted that the combined proportion of severe dehydration of

\begin{tabular}{|c|c|c|c|c|c|c|}
\hline \multirow{2}{*}{$\begin{array}{l}\text { Age-group } \\
\text { (months) }\end{array}$} & \multicolumn{2}{|c|}{ Rotavirus diarrhoea } & \multicolumn{2}{|c|}{ Cholera cases } & \multicolumn{2}{|c|}{$\begin{array}{l}\text { Non-rotavirus and non- } \\
\text { cholera diarrhoea cases }\end{array}$} \\
\hline & No. & $\%$ & No. & $\%$ & No. & $\%$ \\
\hline $0-5$ & 1 & 5.6 & 1 & 3.1 & 1 & 4.2 \\
\hline $6-11$ & 8 & 44.4 & 1 & 3.1 & 2 & 8.4 \\
\hline $12-17$ & 3 & 16.7 & 4 & 12.5 & 3 & 12.5 \\
\hline $18-23$ & 3 & 16.7 & 4 & 12.5 & 4 & 16.7 \\
\hline $24-59$ & 3 & 16.7 & 22 & 68.8 & 14 & 58.3 \\
\hline Total & 18 & 100 & 32 & 100 & 24 & 100 \\
\hline
\end{tabular}




\begin{tabular}{|c|c|c|c|c|c|c|c|c|}
\hline \multirow{3}{*}{$\begin{array}{l}\text { Total no. } \\
\text { of rotavirus } \\
\text { strains }\end{array}$} & \multirow{3}{*}{$\begin{array}{l}\text { Typing of } \\
\text { rotavirus } \\
\text { completed }\end{array}$} & \multirow{3}{*}{ GP type } & \multicolumn{4}{|c|}{ No. of rotavirus strain (\%) } & \multirow{2}{*}{\multicolumn{2}{|c|}{ Total $(\mathrm{n}=114)$}} \\
\hline & & & \multicolumn{2}{|c|}{$\begin{array}{c}\text { Bakerganj } \\
(\mathrm{n}=45)\end{array}$} & \multicolumn{2}{|c|}{$\begin{array}{c}\text { Mathbaria } \\
\quad(n=69)\end{array}$} & & \\
\hline & & & No. & $\%$ & No. & $\%$ & No. & $\%$ \\
\hline \multirow{14}{*}{114} & \multirow{14}{*}{114} & G1 [P8] & 14 & 31.1 & 34 & 49.3 & 48 & 42.1 \\
\hline & & G1 [P6] & 1 & 2.2 & 0 & 0 & 1 & 0.9 \\
\hline & & G2 [P4] & 17 & 37.8 & 26 & 37.7 & 43 & 37.7 \\
\hline & & G2 [P6] & 2 & 4.4 & 0 & 0 & 2 & 1.8 \\
\hline & & G2 [P8] & 1 & 2.2 & 0 & 0 & 1 & 0.9 \\
\hline & & G2 [P0] & 1 & 2.2 & 0 & 0 & 1 & 0.9 \\
\hline & & $\mathrm{G} 2[\mathrm{P}$ mixed] & 1 & 2.2 & 3 & 4.3 & 4 & 3.5 \\
\hline & & G4 [P8] & 1 & 2.2 & 0 & 0 & 1 & 0.9 \\
\hline & & G9 [P6] & 0 & 0 & 1 & 1.4 & 1 & 0.9 \\
\hline & & G9 [P8] & 2 & 4.4 & 3 & 4.3 & 5 & 4.4 \\
\hline & & G9 [P0] & 1 & 2.2 & 0 & 0 & 1 & 0.9 \\
\hline & & G12 [P6] & 2 & 4.4 & 2 & 0 & 4 & 3.5 \\
\hline & & G0 [P6] & 1 & 2.2 & 0 & 0 & 1 & 0.9 \\
\hline & & G0 [P8] & 1 & 2.2 & 0 & 0 & 1 & 0.9 \\
\hline
\end{tabular}

rotavirus and cholera accounted for $68 \%$ (50/74) of the total severe dehydration cases, which was significantly $(\mathrm{p}<0.001)$ higher than that of the 'non-rotavirus and non-cholera' diarrhoea patients $(32 \%$; 24/74).

Further analysis of severe dehydration status by agegroup revealed that under-two children accounted for $83 \%(15 / 18)$ of severe dehydration among the rotavirus group (Table 3 ). This was significantly $(\mathrm{p}<0.01)$ higher than the proportion $(31 \% ; 10 / 32)$ observed among the cholera patients and was also higher $(\mathrm{p}<0.05)$ than the proportion $(42 \%$; $10 / 24$ ) of the 'non-rotavirus and non-cholera' patients. Further, the highest proportion (44\%; $8 / 18$ ) of severely-dehydrated rotavirus-infected children was found in the age-group of 6-11 months. This age-group accounted for the largest proportion of rotavirus-positive patients.

All the 114 rotavirus-positive samples were analyzed to determine the circulating rotavirus strains (G serotype and P genotype) in our rural surveillance sites. Analysis of strains revealed that the most frequently-identified strains were G1 P[8] (42\%) and G2 P[4] (38\%) (Table 4). These two strains accounted for about $80 \%$ of the identified circulating strains in our two surveillance sites. A number of other $\mathrm{G}$ and $\mathrm{P}$ strains were also identified, including G9 P[8] (4\%) and G12 P[6] (4\%).

Analysis of the distribution of rotavirus-associated diarrhoea by months did not indicate distinct seasonal patterns in any of our surveillance sites com- pared to cholera, which showed clear seasonality. However, rotavirus was isolated almost throughout the year.

\section{DISCUSSION}

Over the last few decades, remarkable improvements have been made in understanding the epidemiology, ecology, interventions, and prevention methods of diarrhoeal diseases. However, the incidence of diarrhoea appears to have remained stable globally (21). The present study was conducted in rural UHCs which are the grassroots-level rural health facilities that represent the mainstay for providing both outpatient and inpatient care for nearly $90 \%$ of the rural population of Bangladesh.

Our study has revealed that rotavirus infection was responsible for about $25 \%$ of the under-five children seeking treatment for acute watery diarrhoea at the rural health facilities. This was higher than observed previously (20\%) in urban health facilities of Bangladesh (22). Our observation, however, is almost similar to that (27-36\%) of the more recent study in rural Matlab (16). It should be noted that the ICDDR,B treatment centres in rural Matlab provide treatment exclusively for patients with diarrhoea and diarrhoea-associated illness. By contrast, the health facilities where our study was conducted provided treatment for all types of illness. Our study revealed that $77 \%$ of the rotavirus-infected patients were aged less than two years. Similar observations have been documented in a few other countries $(23,24)$. 
In the recent decades, the proportion of hospitalizations due to diarrhoea attributable to rotavirus is thought to have increased (25). This study confirms the view that, in Bangladesh, diarrhoeal disease is one of the main causes of hospitalizations of children. In rural health facilities, children with dehydration, including those having signs of some dehydration, are usually hospitalized. We have shown that, of all the under-five children hospitalized for various types of illness, 38\% were admitted due to acute watery diarrhoea. This has not been previously documented in Bangladesh. We have also demonstrated that diarrhoea due to rotavirus accounted for $13 \%$ (range 12-14\%) of all hospitalizations in under-five children in the two rural areas. This also, to our knowledge, has not been previously reported in Bangladesh.

On a global scale, 500,000-600,000 infants and young children die from rotavirus-associated diarrhoea annually (4) due to limited access to healthcare facilities. Assessment of diarrhoeal deaths was not within the scope of this study. There were no deaths among the patients who attended our surveillance health facilities as prompt and adequate treatment was provided to them. It is known that diarrhoea patients with severe dehydration are at a higher risk of death if not promptly treated with intravenous fluid compared to patients having some dehydration, which can be corrected mostly with oral rehydration therapy.

A recent study in Matlab, Bangladesh, reported that $20 \%$ of rotavirus-infected children had signs of some dehydration, and only $0.3 \%$ had severe dehydration (16). This finding did not accord with the widely-accepted view that diarrhoea due to rotavirus is one of the major contributors to deaths in young children, particularly in developing countries. In contrast, our study revealed that over $47 \%$ of the rotavirus-affected children had signs of some dehydration, and about $16 \%$ were severely dehydrated. The findings of our study suggest that assessment of dehydration status based on pooled data of under-five children does not reflect the true proportion of age-specific severe dehydration; thus, the risk of death is involved. We have shown that the use of conventional grouping 'aged less than five years' revealed that $16 \%$ of rotavirus-infected children had signs of severe dehydration. This prompted us to look further into age-specific distribution of severe dehydration, which revealed that rotavirus-infected under-two children accounted for more than $80 \%$ of the severely-dehydrated patients. A study in Mainland China reported similar findings (26). The highest (44\%) proportion of age-specific severe dehydration in our study was observed in children aged less than 6 months to
11 months. This observation contributes to the notion that children in this age-group are at a higher risk of death from diarrhoea due to rotavirus. The findings of our study suggest that rotavirus and cholera are the two major contributors to severe dehydration associated with acute watery diarrhoea; thus, the risk of death is involved, particularly in under-five children in rural areas of Bangladesh. It is known that enterotoxigenic Escherichia coli (ETEC) is also one of the major causes of acute watery diarrhoea, particularly in undertwo children (27). However, as mentioned earlier, ETEC was beyond the scope of the current study.

In several countries, rotavirus tends to occur in cooler months (24). In Bangladesh, a higher incidence has been reported during the winter and monsoon months in the middle-belt regions (2). However, in our study sites, there were no distinct seasonal patterns of incidence of rotavirus. This may have been due to locations of our surveillance sites. Our study sites are situated in the southern coastal region of the country where the duration of cooler months is relatively much shorter, and the region is warmer than the middle-belt and northern areas of the country.

A study in Dhaka and Matlab, during 2005-2006, reported that G2 P[4] (43\%) and G12 P[6] (11\%) were the two most-prevalent circulating strains (2). By contrast, we observed that G1 P[8] (42\%) and G2 P [4] (38\%) were the most frequently-circulating strains in our surveillance sites in southern Bangladesh. The proportion of G12 P[6] strains found in our study sites was much lower (4\%) than that observed in the earlier study (2). The difference in the distribution of serotypes isolated from different regions of the country suggests that circulating serotype within a country may have geographical variations.

Our study was limited to only one of the several geo-ecological regions of the country; therefore, this does not reflect the true magnitude of the disease in Bangladesh. However, we have demonstrated that about $25 \%$ of the under-five children seeking treatment for acute watery diarrhoea in rural health facilities were infected with rotavirus. Implication of this, on a national scale, will be significant given that acute watery diarrhoea is the leading cause of hospitalizations of under-five children in rural hospitals in Bangladesh (28).

Initiatives are underway for the introduction of rotavirus vaccine in countries where diarrhoea due to rotavirus is a public-health concern (29). However, efforts will be needed to improve the effectiveness of the vaccine and to use it in countries where it 
will be most beneficial. Ideally, these requirements can be achieved by implementing national surveillance systems for rotavirus which are efficient and reliable. Bangladesh qualifies on the priority list of countries for the introduction of rotavirus vaccine. Therefore, information-collection methods which will provide relatively reliable data, such as distribution of the disease in different geographical regions of the country, including seasonality and rate of hospitalization, need to be established.

\section{ACKNOWLEDGEMENTS}

This research was funded by the Programme for Appropriate Technology in Health (PATH) (Grant No. GAV.1142-01-07231-LPS) and the US National Institutes of Health (NIH) (Grant No. 1R01A13912901). ICDDR,B acknowledges with gratitude the commitment of PATH and NIH to the Centre's research efforts.

The abstract of the study was presented at the $12^{\text {th }}$ Asian Conference on Diarrhoeal Diseases and Nutrition (12 $2^{\text {th }}$ ASCODD) held in Yogyakarta, Indonesia, from 25 to 27 May 2009.

Declaration of Interest: There was no conflict of interest.

\section{REFERENCES}

1. Parashar UD, Gibson CJ, Bresse JS, Glass RI. Rotavirus and severe childhood diarrhoea. Emerg Infect Dis 2006;12:304-6.

2. Rahman M, Sultana R, Ahmed G, Nahar S, Hassan ZM, Saiada F et al. Prevalence of G2P[4] and G12P[6] rotavirus, Bangladesh. Emerg Infect Dis 2007;13:1824.

3. Broor S, Ghosh D, Mathur P. Molecular epidemiology of rotavirus in India. Indian J Med Res 2003;118:5967.

4. The WHO position paper, rotavirus vaccine. $W k l y$ Epidemiol Rec 2007;82:285-96.

5. Miller MA, McCann L. Policy analysis of the use of hepatitis B, Haemophilus influenzae type b-, Streptococcus pneumoniae-conjugate and rotavirus vaccines in national immunization schedules. Health Econ 2000;9:19-35.

6. Molbak K, Fischer-Perch TK, Mikkelsen CS. The estimation of mortality due to rotavirus infections in sub-Saharan Africa. Vaccine 2001;19:393-5.

7. Parashar UD, Hummelman EG, Bresee JS, Miller MA, Glass RI. Global illness and deaths caused by rotavirus disease in children. Emerg Infect Dis 2003;9:56572.

8. Velazquez FR, Matson DO, Calva JJ, Guerrero ML, Morrow AL, Campbell SC et al. Rotavirus infection in infants as protection against subsequent infections. N Engl J Med 1996;335:1022-8.
9. Black RE, Lopez de Romaña G, Brown KH, Bravo N, Bazalar OG, Kanashiro HC. Incidence and etiology of infantile diarrhea and major routes of transmission in Huascar, Peru. Am J Epidemiol 1989;129:785-99.

10. Cravioto A, Reyes RE, Trujillo F, Uribe F, Navarro A, De La Roca JM et al. Risk of diarrhea during the first year of life associated with initial and subsequent colonization by specific enteropathogens. Am J Epidemiol 1990;131:886-904.

11. Black RE, Greenberg HB, Kapikian AZ, Brown KH, Becker S. Acquisition of serum antibody to Norwalk virus and rotavirus and relation to diarrhea in a longitudinal study of young children in rural Bangladesh. J Infect Dis 1982;145:483-9.

12. Zaki AM, DuPont HL, el Alamy MA, Arafat RR, Amin $\mathrm{K}$, Awad MM et al. The detection of enteropathogens in acute diarrhea in a family cohort population in rural Egypt. Am J Trop Med Hyg 1986;35:1013-22.

13. Tsai $\mathrm{CH}$, Chiu HH, Abe T. Epidemiologic features of rotavirus infection in Taiwan: a review. Pediatr Int 2000;42:411-4.

14. Punyaratabandhu P, Vathanophas K, Varavithya W, Sangchai R, Athipanyakom S, Echeverria P et al. Childhood diarrhoea in a low-income urban community in Bangkok: incidence, clinical features, and child caretaker's behaviours. J Diarrhoeal Dis Res 1991;9:244-9.

15. Unicomb LE, Kilgore PE, Faruque ASG, Hamdani JD, Fuchs GJ, Albert MJ et al. Anticipating rotavirus vaccines: hospital-based surveillance for rotavirusassociated diarrhea and estimate of disease burden in Bangladesh. Pediatr Infec Dis J 1997;16:947-51.

16. Zaman K, Yunus M, Faruque ASG, El Arifeen S, Hossain I, Azim T et al. S urveillance of rotavirus in a rural diarrhoea treatment centre in Bangladesh, 2000-2006. Vaccine 2009;27:F31-4.

17. Sack RB, Siddique AK, Longini IM, Jr., Nizam A, Yunus M, Islam MS et al. A 4-year study of the epidemiology of Vibrio cholerae in four rural areas of Bangladesh. J Infect Dis 2003;187:96-101.

18. World Health Organization. Guidelines for cholera control. Rev. 1992. Geneva: World Health Organization, 1992.53 p. (WHO/CDD/SER/80.4 Rev.4 (1992)).

19. World Health Organization. Vaccine and biologicals. Generic protocols for (i) hospital-based surveillance to estimate the burden of rotavirus gastroenteritis in children and (ii) a community-based survey on utilization of health care services for gastroenteritis in children. Geneva: World Health Organization, 2002. 67 p. (WHO/V\&B/02.15).

20. World Health Organization. Manual for laboratory investigation of acute enteric infections. Geneva: 
World Health Organization, 1987. 111 p. (WHO/ CDD/83.3/rev.1).

21. Kosek M, Bern C, Guerrant RL. The global burden of diarrhoeal disease, as estimated from studies published between 1992 and 2000. Bull World Health Organ 2003;81:197-204.

22. Unicomb LE, Kilgore PE, Faruque ASG, Hamdani JD, Fuchs GJ, Albert MJ et al. Anticipating rotavirus vaccines: hospital-based surveillance for rotavirus diarrhea and estimate of disease burden in Bangladesh. Pediatr Infec Dis J 1997;16:947-51.

23. Flem ET, Kasymbekova KT, Vainio K, Gentsch J, Abdikarimov ST, Glass RI et al. Rotavirus infection in hospitalized children and estimates of disease burden in Kyrgyzstan, 2005-2007. Vaccine 2009;27:F35-9.

24. Kim JS, Kang JO, Cho SC, Jang YT, Min SA, Park $\mathrm{TH}$, et al. Epidemiological profile of rotavirus infection in the Republic of Korea: results from prospective surveillance in the Jeongeub district, 1 July 2002 through 30 June 2004. J Infect Dis 2005;192:S49-56.

25. Bresee JS, Hummelman E, Nelson EA, Glass RI. Rota- virus in Asia: the value of surveillance for informing decisions about the introduction of new vaccines. $J$ Infect Dis 2005;192:S1-5.

26. Li DD, Liu N, Yu JM, Zhang Q, Cui SX, Zhang DI et al. Molecular epidemiology of G9 rotavirus strains in children with diarrhoea hospitalized in Mainland China from January 2006 to December 2007. Vaccine 2009;27:F40-5.

27. Qadri, F, Saha A, Ahmed T, Al Tarique A, Begum YA, Svennerholm AM. Disease burden due to enterotoxigenic Escherichia coli in the first 2 years of life in an urban community in Bangladesh. Infect Immun 2007;75:3961-8.

28. Ahmed S, Siddique AK, Iqbal A, Rahman FK, Islam $\mathrm{MN}$, Sobhan MA et al. Causes of hospitalizations at upazilla health complexes in Bangladesh. J Health Popul Nutr 2010;28:399-404.

29. Nelson EA, Widdowson MA, Kilgore PE, Steele D, Parashar UD. A decade of the Asian Rotavirus Surveillance Network: achievements and future directions. Vaccine 2009;27:F1-3. 